Research Paper

\title{
Prognostic value of the serum apolipoprotein $B$ to apolipoprotein A-I ratio in metastatic colorectal cancer patients
}

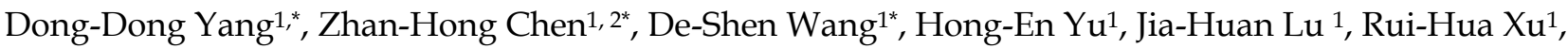 \\ Zhao-Lei Zeng1 ${ }^{\bowtie}$
}

1. Sun Yat-sen University Cancer Center, State Key Laboratory of Oncology in South China, Collaborative Innovation Center for Cancer Medicine, 651 Dong fengdong Road, Guangzhou, 510060, China.

2. Department of Medical Oncology and Guangdong Key Laboratory of Liver Disease, the Third Affiliated Hospital of Sun Yat-sen University, Guangzhou, China.

*These authors contributed equally to this work.

$\square$ Corresponding author: Zhao-Lei Zeng, Address: Sun Yat-sen University Cancer Center, State Key Laboratory of Oncology in South China, Collaborative Innovation Center for Cancer Medicine, 651 Dongfengdong Road, Guangzhou 510060, China. Tel: +86-20-87342297.Fax: +86-20-87343392.E-mail: zengzhl@sysucc.org.cn.

() The author(s). This is an open access article distributed under the terms of the Creative Commons Attribution License (https://creativecommons.org/licenses/by/4.0/). See http:/ /ivyspring.com/terms for full terms and conditions.

Received: 2019.04.11; Accepted: 2019.11.16; Published: 2020.01.01

\begin{abstract}
Background: The aim of our research was to assess the prognostic value of the apolipoprotein $B$ $(A p \circ B)$ to apolipoprotein $A-I$ (ApoA-I) ratio (ApoB/ApoA-I) in metastatic colorectal cancer (mCRC) patients.

Methods: We randomly assigned 838 patients into the training cohort $(n=578)$ and the validation cohort $(n=260)$. The cut-off value of the ApoB/ApoA-I in the training cohort identified by a receiver operating characteristic (ROC) curve was 0.69 and was further validated in the validation cohort. $A$ propensity score matching (PSM) analysis was carried out to eliminate the imbalance in the baseline characteristics of the high and low ApoB/ApoA-I group. The PSM cohort of $542 \mathrm{mCRC}$ patients was generated. We also validated our main findings and conclusions with an independent cohort $(n=150)$. Univariate and multivariate analyses were conducted to explore the independent prognostic value of the ApoB/ApoA-I in the training cohort $(n=578)$, the validation cohort $(n=260)$, the PSM cohort $(n=542)$ and the independent cohort $(n=150)$.

Results: Patients in the high ApoB/ApoA-I group had significantly shorter overall survival compared to those in the low ApoB/ApoA-I group in the training cohort, the validation cohort, the PSM cohort and the independent cohort $(P<0.01)$. Multivariate analysis indicated that the ApoB/ApoA-I was an independent prognostic index for OS in the training cohort [hazard ratio (HR):1.371; $95 \%$ confidence interval $(\mathrm{Cl}): 1.205-1.870, P=0.045]$, the validation cohort (HR: $1.924 ; 95 \% \mathrm{Cl}$ : 1.360-2.723, P<0.001), the PSM cohort (HR: 1.599; 95\% Cl: 1.287-1.988, $P<0.001$ ) and the independent cohort (HR: 1.949; 95\% Cl: 1.014-3.747, $P=0.046$ ).

Conclusions: An increased baseline serum ApoB/ApoA-I is an independent prognostic factor for a poor prognosis in $\mathrm{mCRC}$ patients.
\end{abstract}

Key words: ApoB to ApoA-I ratio, metastatic colorectal cancer, overall survival, prognostic factor, retrospective study.

\section{Introduction}

Colorectal cancer (CRC) was the fourth most common malignant cancer and the second leading cause of cancer-related deaths worldwide in 2018. More than $20 \%$ of CRC patients were diagnosed with 
distant metastasis at initial diagnosis. Although obvious progress was achieved in the fields of molecular targeted therapy, immunotherapy and multidisciplinary treatment (MDT), the 5-year overall survival (OS) rate of metastatic colorectal cancer (mCRC) is still below 20\% [1-3]. Different mCRC patients may have different prognoses, with OS varying from several months to several years. Factors such as liver oligometastasis, RAS mutation and BRAF mutation status have been reported to be prognostic of OS and can also guide treatment options for mCRC patients. The identification of new independent prognostic factors for $\mathrm{mCRC}$ is important and will help us better predict the prognosis of $\mathrm{mCRC}$ patients. Lipid disequilibrium has been reported to contribute to the occurrence and development of CRC. Clinical parameters reflecting lipid disequilibrium may serve as prognostic factors for mCRC patients. An increased low-density lipoprotein cholesterol (LDL-C) to high-density lipoprotein cholesterol (HDL-C) ratio (LHR) has been reported to predict a poor prognosis for mCRC patients with elevations in LDL-C [4-6]. However, little is known about the prognostic value of the $A p o B$ to ApoA-I ratio (ApoB/ApoA-I) in mCRC patients.

Apolipoproteins have been reported to be extensively involved in the processes of cancer occurrence, progression and treatment [7-10]. Several studies have suggested that apolipoproteins can participate in carcinogenesis by promoting proliferation and invasion, enhanced antitumor immunity or drug delivery, and an immediate oxidative stress reaction[11-16]. Apolipoprotein A-I (ApoA-I), mainly synthesized in the liver and the small intestine, is a major fraction of HDL-C, accounting for approximately $70 \%[17,18]$. In CRC patients, the serum ApoA-I level has been reported to be an independent prognostic indicator to predict chemotherapy efficacy and survival time [19-23]. On the other hand, apolipoprotein B (ApoB), an important blood apolipoprotein existing mostly in LDL-C and very-low-density lipoprotein cholesterol (VLDL-C), has been paid less attention to in CRC than ApoA-I. Päivi Sirniö et al. analyzed the correlation between the serum ApoB/ApoA-I and survival in 144 CRC patients. They found that a decreased ApoB/ApoA-I was associated with improved cancer-specific survival and OS. However, the study sample size was small, and the independent prognostic value of the ApoB/ApoA-I was not explored. Furthermore, there were only 19 CRC patients with distant metastasis [20]. Whether the ApoB/ApoA-I can serve as an independent prognostic factor of OS in $\mathrm{mCRC}$ patients remains unknown. We examined a large cohort of $838 \mathrm{mCRC}$ patients to analyze the prognostic value of the pretreatment serum ApoB/ApoA-I on OS in mCRC patients. We also used the propensity score matching method to reduce the imbalance in the baseline characteristics to better identify the independent prognostic value of the ApoB/ApoA-I. Finally, we validated our main findings and conclusions with an independent cohort of $150 \mathrm{mCRC}$ patients.

\section{Methods}

\section{Patient selection and data collection}

We retrospectively collected the clinical data of 1049 patients who were pathologically diagnosed with mCRC between Jan 2005 and Dec 2013 at Sun Yat-sen University Cancer Center (SYSUCC). The inclusion criteria of this study were as follows: 1) Eastern Cooperative Oncology Group performance status (ECOG PS) score $\leq 2,2$ ) received multidisciplinary treatment (MDT), and 3) available complete lab-based serum indexes before treatment. Exclusion criteria were as follows: 1) with previous or coexisting malignant tumors, 2) suffered from acute illnesses, including hepatic failure, severe trauma or stroke, 3) taking lipid-lowering drugs (statins, fibrates), antidiabetic drugs (oral antidiabetics, insulin) or corticosteroids, and 4) without complete follow-up data. We obtained the clinical data of participants, including patient demographics, clinicopathological information (tumor grade, primary tumor site, etc.) and clinical therapy (surgery, chemotherapy regimen, etc.) at first diagnosis from the patient electronic medical record (EMR) system. Serological biomarkers, including lipid metabolism indexes, carcinoembryonic antigen (CEA), and carbohydrate antigen 19-9 (CA19-9), were analyzed by a blood automatic biochemical analyzer. The patients were followed up every 3-6 months until death or dropout, with a median follow-up duration of 26 months (interquartile range (IQR): 15-45 months). A total of 838 patients (534 males, 304 females) were enrolled in our cohort. Then, we randomly divided 838 patients into two independent cohorts, namely, the training cohort $(\mathrm{N}=578)$ and the validation cohort $(\mathrm{N}=260)$.

An independent cohort of $150 \mathrm{mCRC}$ patients hospitalized in SYSUCC between Jan 2014 and Dec 2014 was also included in this study to validate the independent prognostic value of ApoB/ApoA-I on OS in mCRC patients. 
Patients diagnosed with metastatic colorectal cancer between Jan 2005 and Dec 2013 at Sun Yat-sen University Cancer Center $(n=1049)$

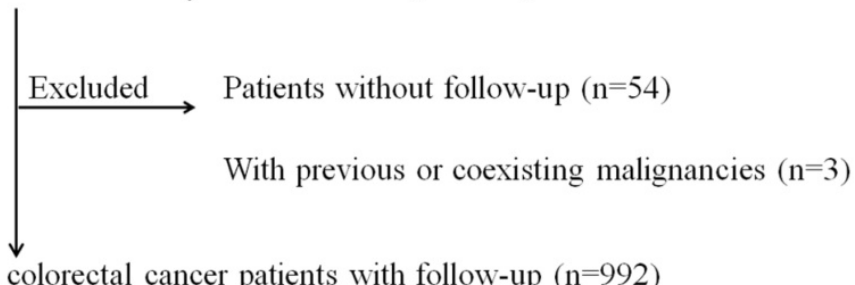

Metastatic colorectal cancer patients with follow-up ( $\mathrm{n}=992)$

$$
\underset{\text { Excluded }}{\longrightarrow} \text { Declined treatment }(\mathrm{n}=4)
$$

Whose ECOG PS score $\geq 3(\mathrm{n}=8)$

With hepatic failure, severe trauma or stroke $(n=6)$

Only received support therapy or hospice care $(n=38)$

Only received one or two cycles chemotherapy $(n=43)$

Taking lipid-lowering or antidiabetic drugs, corticosteroids $(n=45)$

Without avialable records of a blood biochemical test before treatment $(n=10)$

Patients enrolled in the final analyses $(n=838)$

Figure 1. The study flow chart. "ECOG PS score: Eastern Cooperative Oncology Group performance status score.

\section{Statistical analyses}

According to clinical practice, we defined cholesterols $>6.47 \mathrm{mmol} / \mathrm{L}$, triglycerides $>1.70$ $\mathrm{mmol} / \mathrm{L}$, HDL-C $<0.78 \mathrm{mmol} / \mathrm{L}$, LDL-C $>3.40$ mmol/L, ApoA-I <1.05 ng/L, and ApoB >1.15 ng/L as dyslipidemia groups. We defined the best cut-off value of LHR at 2.55 and the ApoB/ApoA-I at 0.69 to stratify patients according to receiver operating characteristic (ROC) curve analysis in the training cohort. The cut-off values of LHR and the ApoB/ApoA-I were further validated in the validation cohort $(\mathrm{N}=260)$, the propensity score matching cohort (PSM) cohort $(\mathrm{N}=542)$ and the independent cohort $(\mathrm{N}=150)$. In this study, we explored the prognostic values of the serum lipid profiles of mCRC patients. A propensity score matching method was used to balance the baseline characteristics in the high and low ApoB/ApoA-I groups. The propensity scores of all patients were estimated by a logistic regression model using the following baseline characteristics as covariates: age, gender, ECOG PS score, primary tumor site, tumor grade, number of metastatic organs, surgery, chemotherapy, CEA and CA19-9. A one-to-one nearest neighbor matching algorithm with an optimal caliper of 0.2 without replacement was used to generate 542 patients, which were named the PSM cohort.

Differences in categorical variables or medians between groups were compared by the chi-square test and the Mann-Whitney test. Kaplan-Meier curves (log-rank test) and Cox proportional hazard regression models (backward method) were used to identify the independent prognostic factors of OS.

All statistical analyses were performed by using MedCalc (version 15.8; MedCalc Software bvba, Acacialaan, Belgium), SPSS (version 24.0; IBM Corp., Armonk, NY, USA) and SAS (version 9.1.3; SAS Institute, Inc., Cary, NC, USA). All statistical tests were two-sided, and $\mathrm{P}$ values less than 0.05 were considered statistically significant.

\section{Results}

\section{Study flow chart and baseline characteristics}

Figure 1 shows the details of the study flow chart. The clinical baseline characteristics of the training cohort $(\mathrm{N}=578)$ and the validation cohort $(\mathrm{N}=260)$ are described in table 1 . We statistically compared the clinical parameters of these two cohorts and found that the two cohorts had comparable baseline characteristics. Patients in the two independent cohorts had similar age and gender 
distributions (training cohort: median age: 54 years, IQR: 43-62 years; gender: $64.4 \%$ males; validation cohort: median age: 55 years, IQR: 44-62 years; gender: $62.3 \%$ males). The primary tumor site was mostly located in the left semicolon (training cohort: $75.1 \%$; validation cohort: $81.2 \%$ ). The proportion of patients who suffered from multiple organ distant metastases was $59.0 \%$ and $56.5 \%$ in the training and validation cohort, respectively. Approximately half of the patients (training cohort: $46.9 \%$; validation cohort: $44.2 \%$ ) in both cohorts received radical or palliative surgery. The serum CEA and CA19-9 profiles were as follows: CEA $>5 \mathrm{ng} / \mathrm{ml}: 67.3 \%$ and CA19-9 $>35$ $\mathrm{ng} / \mathrm{ml}: 50.5 \%$ in the training cohort, CEA $>5 \mathrm{ng} / \mathrm{ml}$ : $70.0 \%$ and CA19-9 $>35 \mathrm{ng} / \mathrm{ml}: 54.2 \%$ in the validation cohort. As displayed in table 1, the number of patients whose serum ApoB/ApoA-I levels were under the cut-off value were $139(24.0 \%)$ and 61 (23.5\%) in the training and validation cohort, respectively.

The results shown in table 2 indicated that we successfully balanced the patients' baseline characteristics with the propensity score matching method, and 542 patients were included in the PSM cohort. The demographic and baseline characteristics of the PSM cohort $(n=542)$ and the independent cohort $(n=150)$ are shown in table 3 . The median age of the PSM cohort was 53 years (IQR: 43-62 years), and most of the patients $(63.8 \%)$ enrolled were male. The proportion of patients who received traditional chemotherapy combined with targeted therapy was $28 \%$. In the PSM cohort, baseline variables, including age, gender, ECOG PS score, primary tumor site, tumor grade, number of metastatic organs, surgery, chemotherapy, CEA and CA19-9, were well balanced in the high ApoB/ApoA-I group and the low ApoB/ApoA-I group (table 2). Thus, the independent prognostic value of the ApoB/ApoA-I on OS in mCRC patients can be better explored.

\section{Survival Analysis}

The endpoint of our research was OS, which was defined as the date from the initial diagnosis to death or the time of the last follow-up. The median OS for 838 patients was 22 months (IQR: 10.0-31.0).

Kaplan-Meier curve analysis indicated that an elevated ApoB/ApoA-I was associated with worse survival in the training cohort, the validation cohort, the PSM cohort and the independent cohort $(P<0.01)$ (Figure 2).

\section{Univariate and multivariate analyses}

In the training cohort, univariate analysis showed that the primary tumor site, tumor grade, tumor resection, CEA, CA19-9, cholesterols, triglycerides, HDL-C, LDL-C, LDL-C/HDL-C and
ApoB/ApoA-I were prognostic of OS (Figures 2A and $3)$.

Table 1. Baseline demographics and clinical characteristics of the training cohort and the validation cohort.

\begin{tabular}{|c|c|c|c|}
\hline Characteristics & $\begin{array}{l}\text { Training Cohort, } \mathrm{n} \\
(\%)\end{array}$ & $\begin{array}{l}\text { Validation Cohort, } \mathrm{n} \\
(\%)\end{array}$ & $P$ value \\
\hline Total & $578(100.0)$ & $260(100.0)$ & \\
\hline $\begin{array}{l}\text { Age, years } \\
\text { (median/IQR) }\end{array}$ & $54(43-62)$ & $55(44-62)$ & 0.805 \\
\hline Gender & & & 0.587 \\
\hline Male & $372(64.4)$ & $162(62.3)$ & \\
\hline Female & 206(35.6) & $98(37.7)$ & \\
\hline ECOG PS score & & & 0.879 \\
\hline 0 & $343(59.3)$ & $162(62.3)$ & \\
\hline $1-2$ & $235(40.7)$ & $98(37.7)$ & \\
\hline Primary tumor site & & & 0.062 \\
\hline Right & $144(24.9)$ & $49(18.8)$ & \\
\hline Left & $434(75.1)$ & $211(81.2)$ & \\
\hline Tumor grade & & & 0.440 \\
\hline G1-2 & $427(73.9)$ & 199(76.5) & \\
\hline G3 & $151(26.1)$ & $61(23.5)$ & \\
\hline $\begin{array}{l}\text { Number of metastatic } \\
\text { organs }\end{array}$ & & & 0.545 \\
\hline 1 & $237(41.0)$ & $113(43.5)$ & \\
\hline$\geq 2$ & $341(59.0)$ & $147(56.5)$ & \\
\hline $\begin{array}{l}\text { Primary/Metastatic } \\
\text { tumor resection }\end{array}$ & & & 0.501 \\
\hline No & $307(53.1)$ & $145(55.8)$ & \\
\hline Yes & $271(46.9)$ & $115(44.2)$ & \\
\hline Chemotherapy & & & 0.281 \\
\hline Chemo & $410(70.9)$ & 194(74.6) & \\
\hline Combination & $168(29.1)$ & $66(25.4)$ & \\
\hline CEA (ng/ml) & & & 0.471 \\
\hline$>5$ & $389(67.3)$ & $182(70.0)$ & \\
\hline$\leq 5$ & $189(32.7)$ & $78(30.0)$ & \\
\hline CA19-9 (ng/ml) & & & 0.332 \\
\hline$>35$ & $292(50.5)$ & $141(54.2)$ & \\
\hline$\leq 35$ & $286(49.5)$ & $119(45.8)$ & \\
\hline $\begin{array}{l}\text { Cholesterols } \\
(\mathrm{mmol} / \mathrm{L})\end{array}$ & & & 0.557 \\
\hline$>6.47$ & $63(10.9)$ & $32(12.3)$ & \\
\hline$\leq 6.47$ & $515(89.1)$ & $228(87.7)$ & \\
\hline $\begin{array}{l}\text { Triglycerides } \\
(\mathrm{mmol} / \mathrm{L})\end{array}$ & & & 0.793 \\
\hline$>1.70$ & $139(24.0)$ & $60(23.1)$ & \\
\hline$\leq 1.70$ & $439(76.0)$ & $200(76.9)$ & \\
\hline HDL-C (mmol/L) & & & 0.664 \\
\hline$\geq 0.78$ & $504(87.2)$ & $224(86.2)$ & \\
\hline$<0.78$ & $74(12.8)$ & $36(13.8)$ & \\
\hline LDL-C (mmol/L) & & & 0.365 \\
\hline$>3.40$ & $236(40.8)$ & $115(44.2)$ & \\
\hline$\leq 3.40$ & $342(59.2)$ & $145(55.8)$ & \\
\hline ApoA-I (ng/L) & & & 0.938 \\
\hline$\geq 1.05$ & $371(64.2)$ & $166(63.8)$ & \\
\hline$<1.05$ & 207(35.8) & $94(36.2)$ & \\
\hline ApoB (ng/L) & & & 0.288 \\
\hline$>1.15$ & $165(28.5)$ & $84(32.3)$ & \\
\hline$\leq 1.15$ & $413(71.5)$ & $176(67.7)$ & \\
\hline LDL-C/HDL-C & & & 0.877 \\
\hline$\geq 2.55$ & $366(63.3)$ & $97(37.3)$ & \\
\hline$<2.55$ & $212(36.7)$ & $163(62.7)$ & \\
\hline ApoB/ApoA-I & & & 0.930 \\
\hline$\geq 0.69$ & $439(76.0)$ & $199(76.5)$ & \\
\hline$<0.69$ & $139(24.0)$ & $61(23.5)$ & \\
\hline \multicolumn{4}{|c|}{$\begin{array}{l}\text { IQR: interquartile range; ECOG PS score: Eastern Cooperative Oncology Group } \\
\text { performance status score; CEA: serum carcinoembryonic antigen; CA19-9: } \\
\text { carbohydrate antigen 19-9; HDL-C: high-density lipoprotein cholesterol; LDL-C: } \\
\text { low-density lipoprotein cholesterol; ApoA-I: apolipoprotein A-I; ApoB: } \\
\text { apolipoprotein B; LDL-C/HDL-C: LDL-C to HDL-C ratio; ApoB/ ApoA-I: ApoB to } \\
\text { ApoA-I ratio. }\end{array}$} \\
\hline
\end{tabular}


Table 2. Association analysis of colorectal cancer patients before propensity score matching $(n=838)$ and after propensity score matching $(n=542)$.

\begin{tabular}{|c|c|c|c|c|c|c|}
\hline \multirow{2}{*}{$\begin{array}{l}\text { Cohort } \\
\text { Characteristics }\end{array}$} & \multicolumn{3}{|c|}{ CRC patients before PSM $(n=838)$} & \multicolumn{3}{|c|}{ CRC patients after PSM $(n=542)$} \\
\hline & ApoB/ApoA-I Low, n (\%) & ApoB/ApoA-I High, n (\%) & $P$-value & ApoB/ApoA-I Low, n (\%) & ApoB/ApoA-I High, n (\%) & $P$-value \\
\hline Total & $200(100 \%)$ & $638(100 \%)$ & & $191(100 \%)$ & $351(100 \%)$ & \\
\hline Age, years & & & 0.030 & & & 0.603 \\
\hline$<55$ & $116(58.0)$ & $314(49.2)$ & & $110(57.6)$ & 194(55.3) & \\
\hline$\geq 55$ & $84(42.0)$ & $324(50.8)$ & & $81(42.4)$ & $157(44.7)$ & \\
\hline Gender & & & 0.111 & & & 0.095 \\
\hline Male & $118(59.0)$ & $416(65.2)$ & & $113(59.2)$ & $233(66.4)$ & \\
\hline Female & $82(41.0)$ & $222(34.8)$ & & $78(40.8)$ & $118(33.6)$ & \\
\hline ECOG PS score & & & 0.365 & & & 0.412 \\
\hline 0 & $126(63.0)$ & $379(59.4)$ & & $119(62.3)$ & $206(58.7)$ & \\
\hline $1-2$ & $74(37.0)$ & $259(40.6)$ & & $72(37.7)$ & $145(41.3)$ & \\
\hline Primary tumor site & & & $<0.001$ & & & 0.110 \\
\hline Right & $65(32.5)$ & $128(20.1)$ & & $72(37.7)$ & $81(23.1)$ & \\
\hline Left & $135(67.5)$ & $510(79.9)$ & & $119(62.3)$ & $270(76.9)$ & \\
\hline Tumor grade & & & 0.911 & & & 0.634 \\
\hline G1-G2 & $150(75.0)$ & $476(74.6)$ & & 144(75.4) & $271(77.2)$ & \\
\hline G3 & $50(25.0)$ & $162(25.4)$ & & $47(24.6)$ & $80(22.8)$ & \\
\hline \multicolumn{2}{|c|}{ Number of metastatic organs } & & 0.569 & & & 0.349 \\
\hline 1 & $87(43.5)$ & $263(41.2)$ & & $83(43.5)$ & $138(39.3)$ & \\
\hline$\geq 2$ & $113(56.5)$ & $375(58.8)$ & & $108(56.5)$ & $213(60.7)$ & \\
\hline \multicolumn{2}{|c|}{ Primary/Metastatic tumor resection } & & 0.599 & & & 0.599 \\
\hline No & $109(54.5)$ & $343(53.8)$ & & $103(53.9)$ & 181(51.6) & \\
\hline Yes & $91(45.5)$ & $295(46.2)$ & & $88(46.1)$ & $170(48.4)$ & \\
\hline Chemotherapy & & & 0.216 & & & 0.265 \\
\hline Chemo & 151(75.5) & $453(71.0)$ & & $143(74.9)$ & $247(70.4)$ & \\
\hline Combination & $49(24.5)$ & $185(29.0)$ & & $48(25.1)$ & 104(29.6) & \\
\hline CEA (ng/ml) & & & 0.013 & & & 0.736 \\
\hline$>5$ & $122(61.0)$ & $449(70.4)$ & & $118(61.8)$ & $222(63.2)$ & \\
\hline$\leq 5$ & $78(39.0)$ & $189(29.6)$ & & $73(38.2)$ & $129(36.8)$ & \\
\hline CA19-9 (ng/ml) & & & $<0.001$ & & & 0.610 \\
\hline$>35$ & $82(41.0)$ & $351(55.0)$ & & $80(41.9)$ & $155(44.2)$ & \\
\hline$\leq 35$ & $118(59.0)$ & $287(45.0)$ & & $111(58.1)$ & $196(55.8)$ & \\
\hline
\end{tabular}

" PSM: propensity score matching; ECOG PS score: Eastern Cooperative Oncology Group Performance Status score; CEA: serum carcinoembryonic antigen; CA19-9: carbohydrate antigen 19-9.

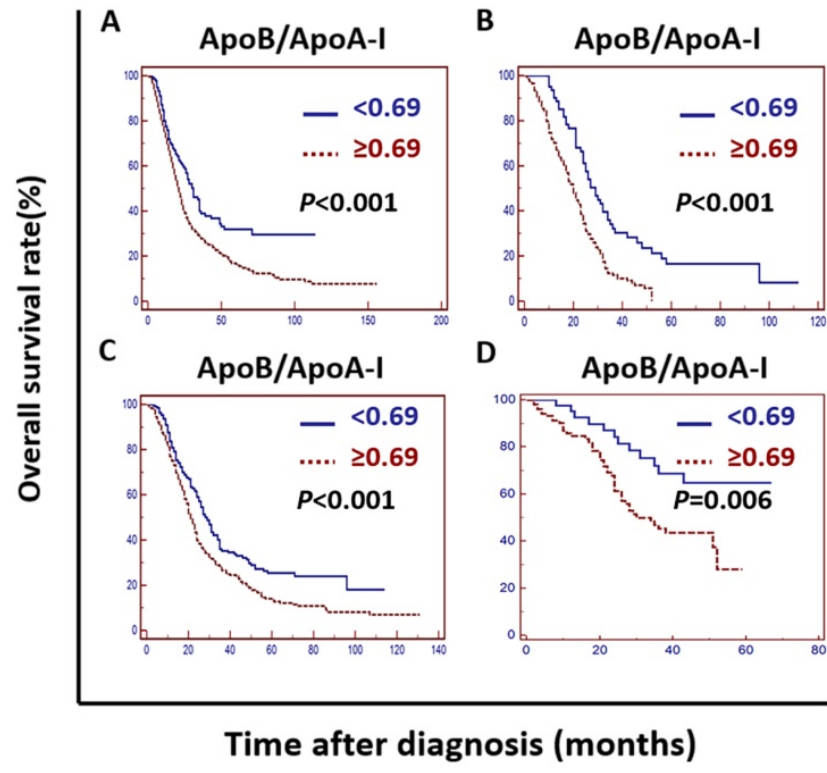

Figure 2. Kaplan-Meier curves depicting OS according to the ApoB/ApoA-I in the training cohort, the validation cohort, the PSM cohort and the independent cohort in patients with metastatic colorectal cancer. A: Kaplan-Meier analysis of OS in the training cohort. B: Kaplan-Meier analysis of OS in the validation cohort. C: Kaplan-Meier analysis of OS in the PSM cohort. D: Kaplan-Meier analysis of OS in the independent cohort.
Table 3. Baseline demographics and clinical characteristics of the propensity score matching (PSM) cohort $(n=542)$ and the independent cohort $(n=150)$.

\begin{tabular}{lll}
\hline Characteristics & PSM cohort, $\mathrm{n}(\%)$ & Independent cohort, $\mathrm{n}(\%)$ \\
\hline Total & $542(100.0)$ & $150(100.0)$ \\
Age, years (median/IQR) & $53(43-62)$ & $61(51-67)$ \\
Gender & & \\
Male & $346(63.8)$ & $88(58.7)$ \\
Female & $196(36.2)$ & $62(41.3)$ \\
ECOG PS score & & \\
0 & $325(60.0)$ & $22(14.7)$ \\
$1-2$ & $217(40.0)$ & $128(85.3)$ \\
Primary tumor site & & \\
Right & $137(25.3)$ & $32(21.3)$ \\
Left & $405(74.7)$ & $118(78.7)$ \\
Tumor grade & & \\
G1-2 & $415(76.6)$ & $112(74.7)$ \\
G3 & $127(23.4)$ & $38(25.3)$ \\
Number of metastatic organs & & \\
1 & $221(40.8)$ & $102(68.0)$ \\
$\geq 2$ & $321(59.2)$ & $48(32.0)$ \\
Primary/Metastatic tumor & & \\
resection & & \\
No & $284(52.4)$ & $43(28.7)$ \\
Yes & $258(47.6)$ & $107(71.3)$ \\
Chemotherapy & & \\
Chemo alone & $390(72.0)$ & $104(69.3)$ \\
Combination & $152(28.0)$ & $46(30.7)$ \\
CEA (ng/ml) & & \\
$>5$ & $340(62.7)$ & $105(70.0)$ \\
$\leq 5$ & $202(37.3)$ & $45(30.0)$ \\
\hline & &
\end{tabular}




\begin{tabular}{|c|c|c|}
\hline \multicolumn{3}{|c|}{ CA19-9(ng/ml) } \\
\hline$>35$ & $235(43.4)$ & $67(44.7)$ \\
\hline$\leq 35$ & $307(56.6)$ & $83(55.3)$ \\
\hline \multicolumn{3}{|c|}{ Cholesterols (mmol/L) } \\
\hline$>6.47$ & $45(8.3)$ & $18(12.0)$ \\
\hline$\leq 6.47$ & 497(91.7) & $132(88.0)$ \\
\hline \multicolumn{3}{|c|}{ Triglycerides (mmol/L) } \\
\hline$>1.70$ & $116(21.4)$ & $24(16.0)$ \\
\hline$\leq 1.70$ & $426(78.6)$ & $126(84.0)$ \\
\hline \multicolumn{3}{|c|}{ HDL-C (mmol/L) } \\
\hline$\geq 0.78$ & $481(88.7)$ & $140(93.3)$ \\
\hline$<0.78$ & $61(11.3)$ & $10(6.7)$ \\
\hline \multicolumn{3}{|c|}{ LDL-C $(\mathrm{mmol} / \mathrm{L})$} \\
\hline$>3.40$ & $186(34.3)$ & $53(35.3)$ \\
\hline$\leq 3.40$ & $356(65.7)$ & $97(64.7)$ \\
\hline \multicolumn{3}{|c|}{ ApoA-I (ng/L) } \\
\hline$\geq 1.05$ & $362(66.8)$ & $49(32.7)$ \\
\hline$<1.05$ & $180(33.2)$ & $101(67.3)$ \\
\hline \multicolumn{3}{|c|}{$\mathrm{ApoB}(\mathrm{ng} / \mathrm{L})$} \\
\hline$>1.15$ & $129(23.8)$ & $40(26.7)$ \\
\hline$\leq 1.15$ & $413(76.2)$ & $110(73.3)$ \\
\hline \multicolumn{3}{|c|}{ LDL-C/HDL-C } \\
\hline$\geq 2.55$ & $297(54.8)$ & $92(61.3)$ \\
\hline$<2.55$ & $245(45.2)$ & $58(38.7)$ \\
\hline \multicolumn{3}{|c|}{ ApoB/ApoA-I } \\
\hline$\geq 0.69$ & $351(64.8)$ & $106(70.7)$ \\
\hline$<0.69$ & $191(35.2)$ & $44(29.3)$ \\
\hline \multicolumn{3}{|c|}{$\begin{array}{l}\text { "IQR: interquartile range; ECOG PS score: Eastern Cooperative Oncology Group } \\
\text { performance status score; CEA: serum carcinoembryonic antigen; CA19-9: } \\
\text { carbohydrate antigen 19-9; HDL-C: high-density lipoprotein cholesterol; LDL-C: } \\
\text { low-density lipoprotein cholesterol; ApoA-I: apolipoprotein A-I; ApoB: } \\
\text { apolipoprotein B; LDL-C/HDL-C: LDL-C to HDL-C ratio; ApoB/ApoA-I: ApoB to } \\
\text { ApoA-I ratio. }\end{array}$} \\
\hline
\end{tabular}

In the validation cohort, univariate analysis showed that the tumor grade, CEA, CA19-9, ApoA-I, ApoB, LDL-C/HDL-C and ApoB/ApoA-I were prognostic of OS (Figures $2 \mathrm{~B}$ and 4 ).

In the PSM cohort, univariate analysis showed that the ECOG PS score, primary tumor site, tumor grade, tumor resection, CEA, CA19-9, HDL-C, LDL-C, LDL-C/HDL-C and ApoB/ApoA-I were prognostic of OS (Figures $2 \mathrm{C}$ and 5 ).

The tumor grade, CEA, CA19-9, LDL-C/HDL-C and ApoB/ApoA-I were prognostic of OS in all three cohorts (Figures 3, 4, and 5). Variables significantly prognostic of OS identified by univariate analysis were further analyzed by Cox proportional hazards regression models (backward method) in multivariate analysis. The ApoB/ApoA-I was identified as an independent prognostic factor for OS in all three cohorts [training cohort: hazard ratio (HR): 1.371; 95\% confidence interval (CI): 1.205-1.870, $P=0.045$; validation cohort: HR: 1.924 95\% CI: 1.360-2.723, $P<0.001$; PSM cohort: HR: 1.599 95\% CI: 1.287-1.988, $P<0.001$, tables 4, 5, and 6].

CEA, CA19-9 and tumor grade were also identified as independent prognostic factors for OS in all three cohorts. The LDL-C/HDL-C was identified as an independent prognostic factor for $O S$ in the training cohort. However, the LDL-C/HDL-C was not identified as an independent prognostic factor for OS in the validation cohort or the PSM cohort (Tables 4, 5, and 6).

Univariate and multivariate analyses showed that ApoB/ApoA-I was an independent prognostic factor in the independent cohort (HR: 1.949; 95\% CI: 1.014-3.747, $\mathrm{P}=0.046$ ) (Tables 7).We validated our main findings and conclusions with an independent cohort.

Table 4. Univariate and multivariate analyses of the prognostic factors of overall survival (OS) in the training cohort.

\begin{tabular}{|c|c|c|c|c|c|c|}
\hline \multirow[t]{2}{*}{ Variables } & \multicolumn{3}{|c|}{ Univariate } & \multicolumn{3}{|c|}{ Multivariate } \\
\hline & HR & $95 \% \mathrm{CI}$ & $P$ value & HR & $95 \% \mathrm{CI}$ & $P$-value \\
\hline Age, years $(<55 / \geq 55)$ & 1.198 & $0.986-1.456$ & 0.070 & & & \\
\hline Gender (Male/Female) & 1.044 & $0.853-1.277$ & 0.676 & & & \\
\hline ECOG PS score (0/1-2) & 1.184 & $0.971-1.443$ & 0.095 & & & \\
\hline Primary tumor site ( Left / Right) & 1.477 & $1.185-1.841$ & 0.001 & 1.437 & $1.175-1.757$ & $<0.001$ \\
\hline Tumor grade (G1-2/G3) & 1.410 & $1.128-1.762$ & 0.003 & 1.565 & $1.245-1.966$ & $<0.001$ \\
\hline Number of metastatic organs $(1 / \geq 2)$ & 0.937 & $0.768-1.143$ & 0.521 & & & \\
\hline Primary/Metastatic tumor resection (Yes/No) & 1.443 & $1.185-1.758$ & 0.001 & 1.473 & $1.175-1.757$ & $<0.001$ \\
\hline Chemotherapy (Chemo/combination) & 0.960 & $0.776-1.187$ & 0.705 & & & \\
\hline CEA $(\mathrm{ng} / \mathrm{ml})(<5 / \geq 5)$ & 1.692 & $1.364-2.100$ & $<0.001$ & 1.501 & $1.193-1.889$ & 0.001 \\
\hline CA19-9 (ng/ml) $(<35 / \geq 35)$ & 1.569 & $1.289-1.911$ & $<0.001$ & 1.317 & $1.065-1.630$ & 0.011 \\
\hline Cholesterols $(\mathrm{mmol} / \mathrm{L})(\leq 6.47 />6.47)$ & 1.375 & $1.033-1.8230$ & 0.006 & & & \\
\hline Triglycerides $(\mathrm{mmol} / \mathrm{L})(\leq 1.70 />1.70)$ & 0.789 & $0.625-0.996$ & 0.046 & 0.707 & $0.558-0.896$ & 0.004 \\
\hline HDL-C (mmol/L) $(\geq 0.78 /<0.78)$ & 1.415 & $1.064-1.882$ & 0.017 & & & \\
\hline LDL-C $(\mathrm{mmol} / \mathrm{L})(\leq 3.40 />3.40)$ & 1.266 & $1.041-1.540$ & 0.018 & & & \\
\hline ApoA-I(ng/L) $(\geq 1.05 /<1.05)$ & 1.124 & $0.917-1.378$ & 0.260 & & & \\
\hline $\operatorname{ApoB}(\mathrm{ng} / \mathrm{L})(\leq 1.15 />1.15)$ & 1.123 & $0.984-1.496$ & 0.071 & & & \\
\hline LDL-C/HDL-C $(<2.55 / \geq 2.55)$ & 1.340 & $1.087-1.651$ & 0.006 & 1.334 & $1.109-1.746$ & 0.036 \\
\hline ApoB/ApoA-I $(<0.69 / \geq 0.69)$ & 1.580 & $1.234-2.023$ & $<0.001$ & 1.371 & $1.205-1.870$ & 0.045 \\
\hline
\end{tabular}


Table 5. Univariate and multivariate analyses of the prognostic factors of overall survival (OS) in the validation cohort.

\begin{tabular}{|c|c|c|c|c|c|c|}
\hline \multirow[t]{2}{*}{ Variables } & \multicolumn{3}{|c|}{ Univariate } & \multicolumn{3}{|c|}{ Multivariate } \\
\hline & $\overline{\mathrm{HR}}$ & $95 \% \mathrm{CI}$ & $P$ value & HR & $95 \% \mathrm{CI}$ & $P$-value \\
\hline Age, years $(<55 / \geq 55)$ & 1.029 & $0.774-1.368$ & 0.388 & & & \\
\hline Gender (Male/Female) & 1.044 & $0.853-1.277$ & 0.876 & & & \\
\hline ECOG PS score (0/1-2) & 1.248 & $0.939-1.658$ & 0.127 & & & \\
\hline Primary tumor site ( Left / Right) & 1.072 & $0.749-1.533$ & 0.705 & & & \\
\hline Tumor grade (G1-2/G3) & 1.475 & $1.059-2.056$ & 0.022 & 1.635 & $1.163-2.300$ & 0.005 \\
\hline Number of metastatic organs $(1 / \geq 2)$ & 0.886 & $0.669-1.173$ & 0.397 & & & \\
\hline Primary/Metastatic tumor resection (Yes/No) & 1.087 & $0.822-1.438$ & 0.559 & & & \\
\hline Chemotherapy (Chemo/combination) & 0.970 & $0.706-1.333$ & 0.852 & & & \\
\hline $\operatorname{CEA}(\mathrm{ng} / \mathrm{ml})(<5 / \geq 5)$ & 1.605 & $1.170-2.203$ & 0.003 & 1.441 & $1.029-2.016$ & 0.033 \\
\hline CA19-9 (ng/ml) $(<35 / \geq 35)$ & 1.664 & $1.251-2.214$ & $<0.001$ & 1.489 & $1.101-2.015$ & 0.010 \\
\hline Cholesterols $(\mathrm{mmol} / \mathrm{L})(\leq 6.47 />6.47)$ & 0.955 & $0.618-1.477$ & 0.855 & & & \\
\hline Triglycerides $(\mathrm{mmol} / \mathrm{L})(\leq 1.70 />1.70)$ & 1.208 & $0.872-1.673$ & 0.256 & & & \\
\hline HDL-C $(\mathrm{mmol} / \mathrm{L})(\geq 0.78 /<0.78)$ & 1.424 & $0.947-2.140$ & 0.089 & & & \\
\hline LDL-C $(\mathrm{mmol} / \mathrm{L})(\leq 3.40 />3.40)$ & 1.298 & $0.996-1.726$ & 0.072 & & & \\
\hline ApoA-I (ng/L) $(\geq 1.05 /<1.05)$ & 1.391 & $1.040-1.863$ & 0.026 & & & \\
\hline $\operatorname{ApoB}(\mathrm{ng} / \mathrm{L})(\leq 1.15 />1.15)$ & 1.356 & $1.005-1.861$ & 0.046 & & & \\
\hline LDL-C/HDL-C $(<2.55 / \geq 2.55)$ & 1.491 & $1.107-2.009$ & 0.008 & & & \\
\hline ApoB/ApoA-I $(<0.69 / \geq 0.69)$ & 2.122 & $1.504-2.995$ & $<0.001$ & 1.924 & $1.360-2.723$ & $<0.001$ \\
\hline
\end{tabular}

*ECOG PS score: Eastern Cooperative Oncology Group performance status score; CEA: serum carcinoembryonic antigen; CA19-9: carbohydrate antigen 19-9; HDL-C: high-density lipoprotein cholesterol; LDL-C: low-density lipoprotein cholesterol; ApoA-I: apolipoprotein A-I; ApoB: apolipoprotein B; LDL-C/HDL-C: LDL-C to HDL-C ratio; ApoB/ApoA-I: ApoB to ApoA-I ratio.

A

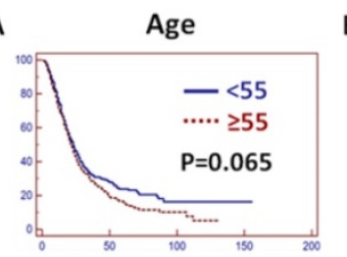

E
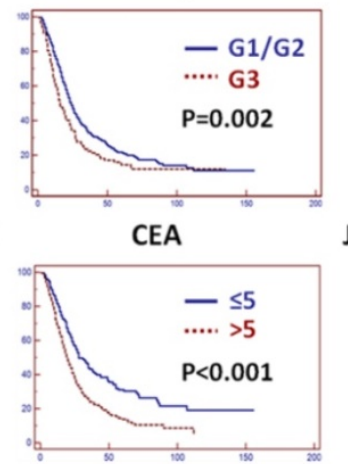

M
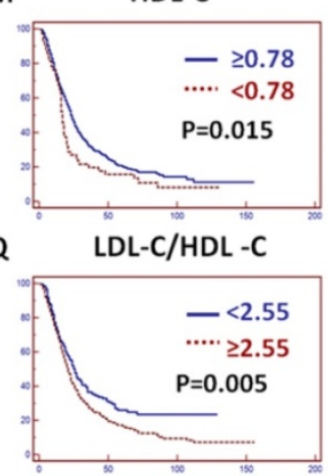

B

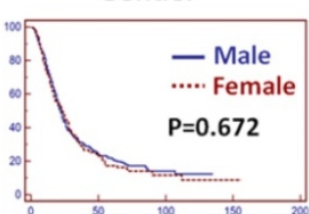

F

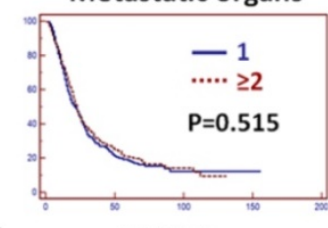

J

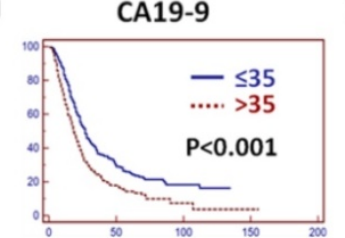

N
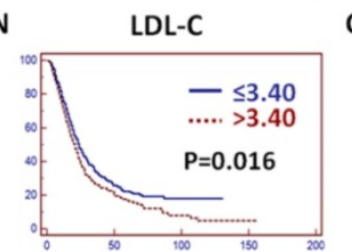

C

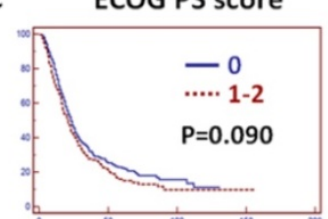

G

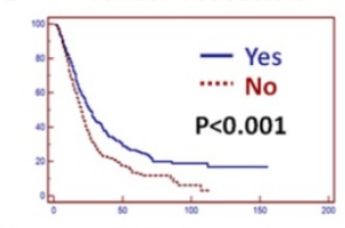

K Cholesterols

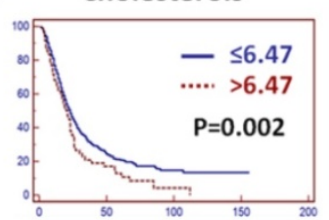

$\circ$

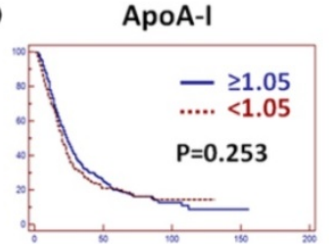

D Primary tumor site

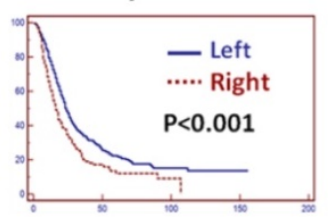

$\mathrm{H}$

Chemotherapy

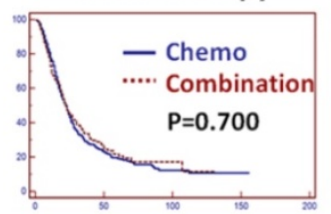

$\mathrm{L}$

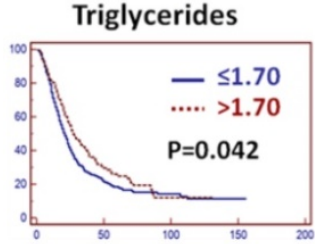

P

ApoB

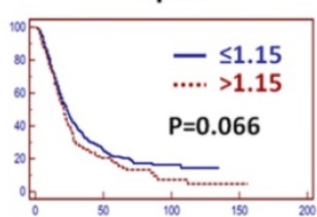

\section{Time after diagnosis(months)}

Figure 3. Kaplan-Meier curves depicting OS according to other factors in the training cohort. *ECOG PS score: Eastern Cooperative Oncology Group performance status score; Metastatic organs: number of metastatic organs; Tumor resection: primary or metastatic tumor resection; CEA: serum carcinoembryonic antigen; CA19-9: carbohydrate antigen 19-9; HDL-C: high-density lipoprotein cholesterol; LDL-C: low-density lipoprotein cholesterol; ApoA-I: apolipoprotein A-I; ApoB: apolipoprotein B; LDL-C/HDL-C: LDL-C to HDL-C ratio. 


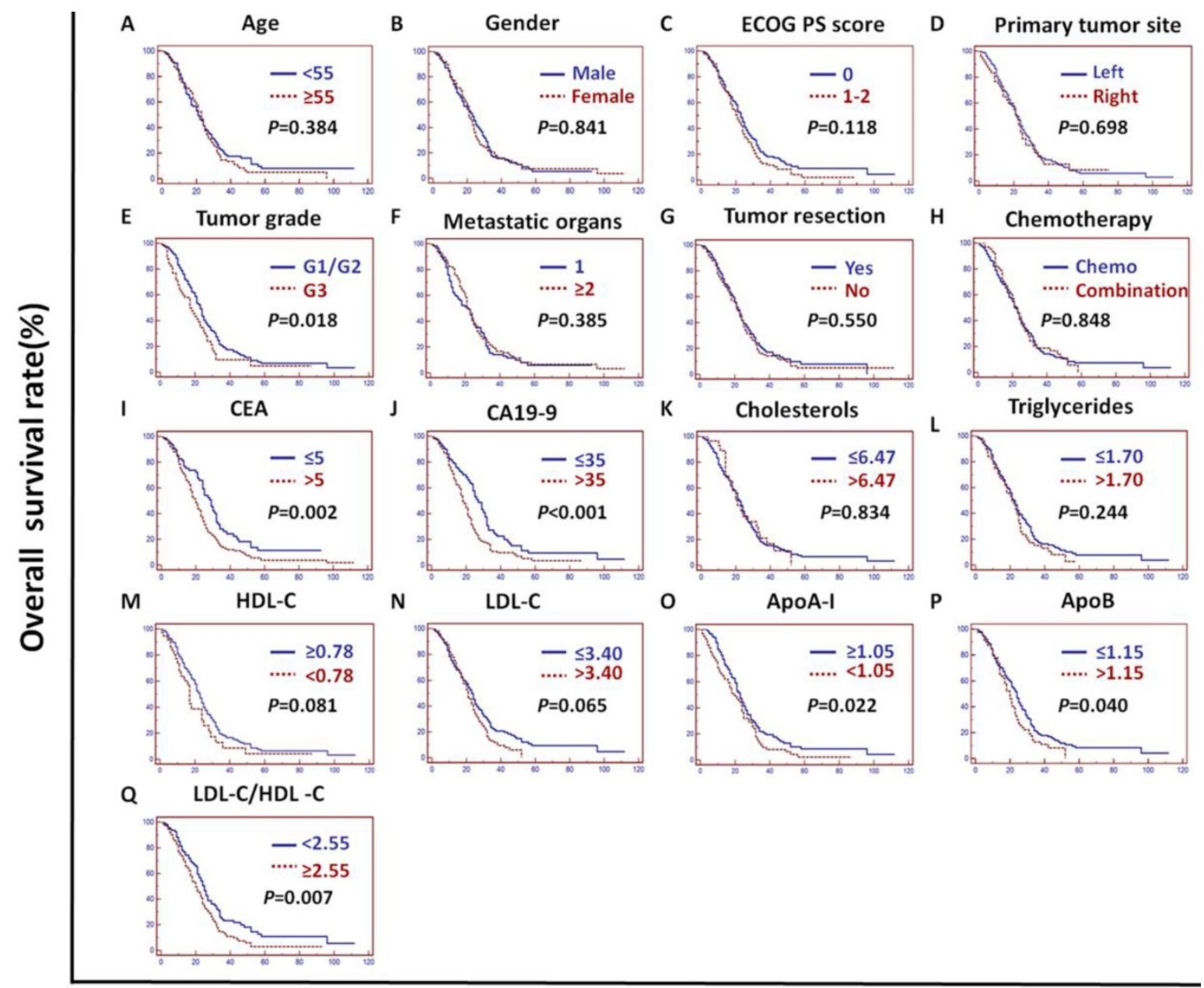

\section{Time after diagnosis(months)}

Figure 4. Kaplan-Meier curves depicting OS according to other factors in the validation cohort. "ECOG PS score: Eastern Cooperative Oncology Group performance status score; Metastatic organs: number of metastatic organs; Tumor resection: primary or metastatic tumor resection; CEA: serum carcinoembryonic antigen; CA19-9:; carbohydrate antigen 19-9; HDL-C: high-density lipoprotein cholesterol; LDL-C: low-density lipoprotein cholesterol; ApoA-I: apolipoprotein A-I; ApoB: apolipoprotein B; LDL-C/HDL-C: LDL-C to HDL-C ratio.

Table 6. Univariate and multivariate analyses of the prognostic factors of overall survival (OS) in the PSM cohort.

\begin{tabular}{|c|c|c|c|c|c|c|}
\hline \multirow[t]{2}{*}{ Variables } & \multicolumn{3}{|c|}{ Univariate } & \multicolumn{3}{|c|}{ Multivariate } \\
\hline & $\overline{\mathrm{HR}}$ & $95 \% \mathrm{CI}$ & $P$ value & HR & $95 \% \mathrm{CI}$ & $P$-value \\
\hline Age, years $(<55 / \geq 55)$ & 1.093 & $0.893-1.399$ & 0.387 & & & \\
\hline Gender (Male/Female) & 1.171 & $0.952-1.440$ & 0.134 & & & \\
\hline ECOG PS score $(0 / 1-2)$ & 1.295 & $1.056-1.588$ & 0.013 & 1.268 & $1.032-1.557$ & 0.024 \\
\hline Primary tumor site ( Left / Right) & 1.531 & $1.207-1.897$ & $<0.001$ & 1.491 & $1.187-1.873$ & 0.001 \\
\hline Tumor grade (G1-2/G3) & 1.445 & $1.141-1.831$ & 0.003 & 1.448 & $1.141-1.836$ & 0.002 \\
\hline Number of metastatic organs $(1 / \geq 2)$ & 0.878 & $0.716-1.078$ & 0.216 & & & \\
\hline Primary/Metastatic tumor resection (Yes/No) & 1.347 & $1.100-1.650$ & 0.004 & 1.309 & $1.066-1.606$ & 0.010 \\
\hline Chemotherapy (Chemo/combination) & 0.957 & $0.767-1.195$ & 0.700 & & & \\
\hline $\operatorname{CEA}(\mathrm{ng} / \mathrm{ml})(<5 / \geq 5)$ & 1.751 & $1.410-2.173$ & $<0.001$ & 1.573 & $1.249-1.981$ & $<0.001$ \\
\hline CA19-9 (ng/ml) $(<35 / \geq 35)$ & 1.721 & $1.401-2.115$ & $<0.001$ & 1.469 & $1.181-1.828$ & 0.001 \\
\hline Cholesterols (mmol/L) $(\leq 6.47 />6.47)$ & 1.109 & $0.779-1.578$ & 0.565 & & & \\
\hline Triglycerides $(\mathrm{mmol} / \mathrm{L})(\leq 1.70 />1.70)$ & 0.814 & $0.634-1.044$ & 0.105 & & & \\
\hline HDL-C $(\mathrm{mmol} / \mathrm{L})(\geq 0.78 /<0.78)$ & 1.413 & $1.034-1.932$ & 0.030 & & & \\
\hline LDL-C $(\mathrm{mmol} / \mathrm{L})(\leq 3.40 />3.40)$ & 1.308 & $1.062-1.612$ & 0.012 & & & \\
\hline ApoA-I (ng/L) $(\geq 1.05 /<1.05)$ & 1.386 & $0.760-2.527$ & 0.287 & & & \\
\hline $\operatorname{ApoB}(\mathrm{ng} / \mathrm{L})(\leq 1.15 />1.15)$ & 1.250 & $0.993-1.574$ & 0.057 & & & \\
\hline LDL-C/HDL-C $(<2.55 / \geq 2.55)$ & 1.299 & $1.059-1.594$ & 0.012 & & & \\
\hline ApoB/ApoA-I $(<0.69 / \geq 0.69)$ & 1.485 & $1.196-1.843$ & $<0.001$ & 1.599 & $1.287-1.988$ & $<0.001$ \\
\hline
\end{tabular}

* PSM cohort: propensity score matching cohort; ECOG PS score: Eastern Cooperative Oncology Group performance status score; CEA: serum carcinoembryonic antigen; CA19-9: carbohydrate antigen 19-9; HDL-C: high-density lipoprotein cholesterol; LDL-C: low-density lipoprotein cholesterol; ApoA-I: apolipoprotein A-I; ApoB: apolipoprotein B; LDL-C/HDL-C: LDL-C to HDL-C ratio; ApoB/ApoA-I: ApoB to ApoA-I ratio. 


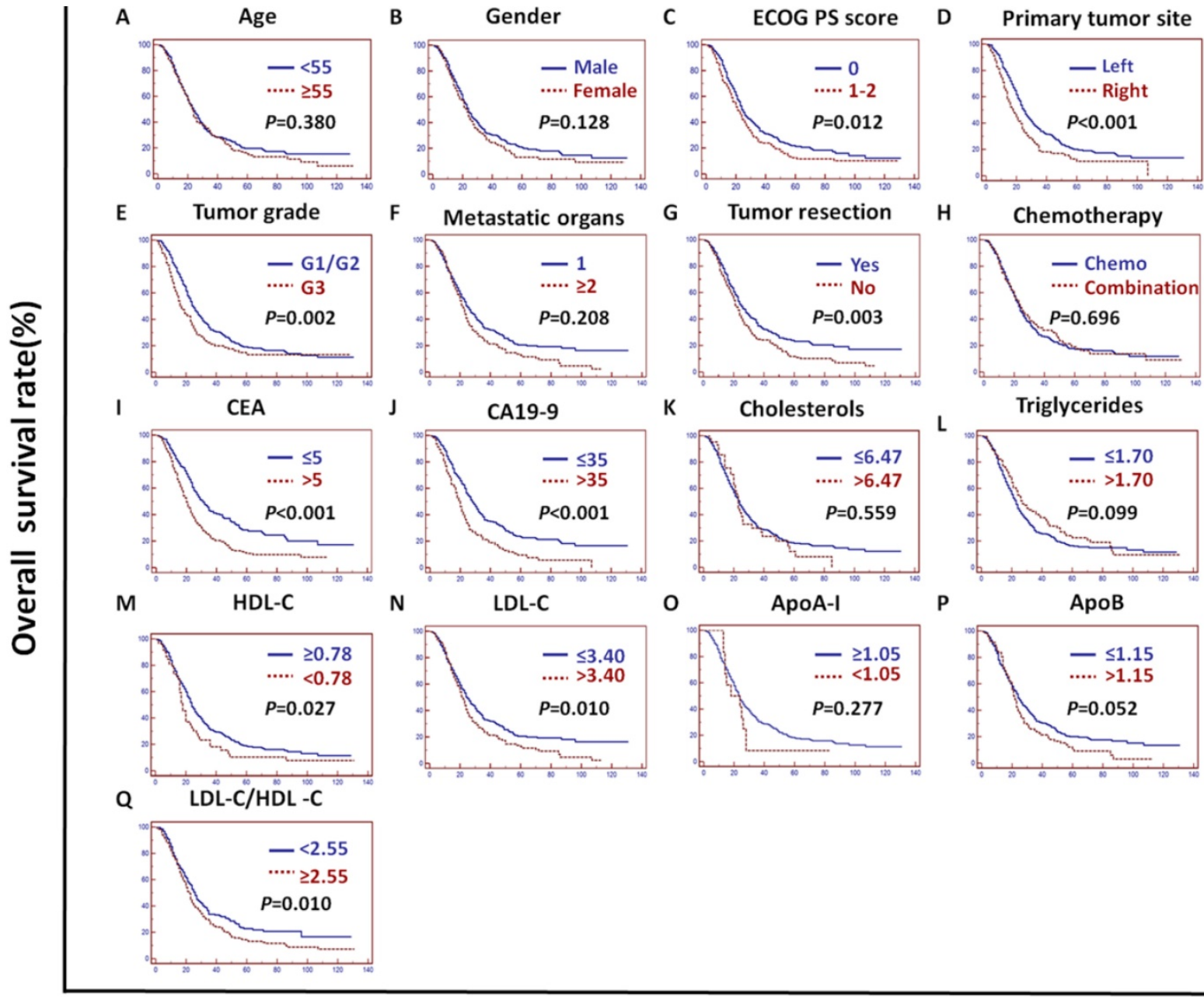

Time after diagnosis(months)

Figure 5. Kaplan-Meier curves depicting OS according to other factors in the PSM cohort. *PSM: propensity score matching; ECOG PS score: Eastern Cooperative Oncology Group performance status score; Metastatic organs: number of metastatic organs; Tumor resection: primary or metastatic tumor resection; CEA: serum carcinoembryonic antigen; CA19-9: carbohydrate antigen 19-9; HDL-C: high-density lipoprotein cholesterol; LDL-C: low-density lipoprotein cholesterol; ApoA-I: apolipoprotein A-I; ApoB: apolipoprotein B; LDL-C/HDL-C: LDL-C to HDL-C ratio.

Table 7. Univariate and multivariate analyses of the prognostic factors of overall survival (OS) in the independent cohort.

\begin{tabular}{|c|c|c|c|c|c|c|}
\hline \multirow[t]{2}{*}{ Variables } & \multicolumn{3}{|c|}{ Univariate } & \multicolumn{3}{|c|}{ Multivariate } \\
\hline & HR & $95 \% \mathrm{CI}$ & $P$ value & HR & $95 \% \mathrm{CI}$ & $P$-value \\
\hline Age, years $(<55 / \geq 55)$ & 1.099 & $0.627-1.925$ & 0.744 & & & \\
\hline Gender (Male/Female) & 0.870 & $0.516-1.466$ & 0.603 & & & \\
\hline ECOG PS score $(0 / 1-2)$ & 0.594 & $0.308-1.144$ & 0.121 & & & \\
\hline Primary tumor site ( Left / Right ) & 0.765 & $0.406-1.440$ & 0.408 & & & \\
\hline Tumor grade (G1-2/G3) & 0.986 & $0.557-1.746$ & 0.961 & & & \\
\hline Number of metastatic organs $(1 / \geq 2)$ & 1.467 & $0.862-2.499$ & 0.160 & & & \\
\hline Primary/Metastatic tumor resection (Yes/No) & 1.970 & $1.154-3.360$ & 0.013 & 1.652 & $0.960-2.843$ & 0.071 \\
\hline Chemotherapy (Chemo/combination) & 1.138 & $0.673-1.925$ & 0.631 & & & \\
\hline $\operatorname{CEA}(\mathrm{ng} / \mathrm{ml})(<5 / \geq 5)$ & 2.474 & $1.327-4.611$ & 0.005 & 1.980 & $1.041-3.766$ & 0.038 \\
\hline CA19-9 (ng/ml) $(<35 / \geq 35)$ & 1.826 & $1.095-3.043$ & 0.022 & & & \\
\hline Cholesterols $(\mathrm{mmol} / \mathrm{L})(\leq 6.47 />6.47)$ & 2.141 & $1.038-4.417$ & 0.040 & & & \\
\hline Triglycerides $(\mathrm{mmol} / \mathrm{L})(\leq 1.70 />1.70)$ & 0.932 & $0.460-1.888$ & 0.845 & & & \\
\hline HDL-C $(\mathrm{mmol} / \mathrm{L})(\geq 0.78 /<0.78)$ & 1.529 & $0.614-3.806$ & 0.365 & & & \\
\hline LDL-C $(\mathrm{mmol} / \mathrm{L})(\leq 3.40 />3.40)$ & 1.215 & $0.709-2.081$ & 0.480 & & & \\
\hline ApoA-I (ng/L) $(\geq 1.05 /<1.05)$ & 1.284 & $0.745-2.213$ & 0.370 & & & \\
\hline ApoB (ng/L) $(\leq 1.15 />1.15)$ & 1.535 & $0.874-2.697$ & 0.138 & & & \\
\hline LDL-C/HDL-C $(<2.55 / \geq 2.55)$ & 1.309 & $0.774-2.215$ & 0.317 & & & \\
\hline ApoB/ApoA-I $(<0.69 / \geq 0.69)$ & 2.391 & $1.264-4.524$ & 0.008 & 1.949 & $1.014-3.747$ & 0.046 \\
\hline
\end{tabular}

* ECOG PS score: Eastern Cooperative Oncology Group performance status score; CEA: serum carcinoembryonic antigen; CA19-9: carbohydrate antigen 19-9; HDL-C: high-density lipoprotein cholesterol; LDL-C: low-density lipoprotein cholesterol; ApoA-I: apolipoprotein A-I; ApoB: apolipoprotein B; LDL-C/HDL-C: LDL-C to HDL-C ratio; ApoB/ApoA-I: ApoB to ApoA-I ratio. 


\section{Discussion}

We confirmed that an increased ApoB/ApoA-I was an independent prognostic factor for shorter OS in $\mathrm{mCRC}$ patients by exploring the prognostic values of clinicopathological variables in the training cohort, the validation cohort, the PSM cohort and the independent cohort. In our research, we explored the independent prognostic value of the ApoB/ApoA-I using the traditional randomization method to generate the training cohort and the validation cohort and using the propensity matching scoring method to generate the PSM cohort. Finally, we validated our main findings and conclusions with an independent cohort.

To the best of our knowledge, we identified for the first time the independent prognostic value of the ApoB/ApoA-I in a large cohort of $\mathrm{mCRC}$ patients.

ApoA-I is an important apolipoprotein that reversely transports cholesterols from peripheral tissues to the liver. It has been reported to be a cofactor for lectin cholesterol acyltransferase (LCAT), which extensively participates in lipid metabolism[24]. In recent decades, many studies have reported the effect of lipid metabolism indexes on cancers, most of which focused on the risk of cancer influenced by lipids or lipoproteins. Several studies have identified that high ApoA-I can sharply increase the risk of breast cancer but inversely reduce the risk of lung cancer $[4,5,7,25-28]$. The prognostic value of serum ApoA-I has been previously investigated, and the results of different studies confirmed that increased ApoA-I is a favorable factor in ovarian cancer, lung cancer, kidney cancer, nonmetastatic nasopharyngeal cancer and $\mathrm{mCRC}$ for OS. A recent retrospective study reported that low serum ApoA-I levels were associated with advanced $\mathrm{T}$ class and TNM stage and systemic inflammation biomarkers in CRC. All of these findings indicate that serum ApoA-I may participate in multiple processes in the occurrence and development of cancers [19, 20, 29-31].

On the other hand, ApoB is known as a carrier that ships lipids, including cholesterols and triglycerides, into extrahepatic tissues. In a large-scale prospective cohort study, the investigators found that high circulating ApoB could increase the risk of CRC in men but reduce the risk of breast cancer in women. Furthermore, elevated blood ApoB can result in increased prevalence rates of CRC and lung cancer [4, 5, 32]. A high ApoB/ApoA-I was defined as a risk factor for atherosclerosis, hypertension and polycystic ovary syndrome (PCOS) [33]. Only one study confirmed that a low ApoB/ApoA-I were favorable prognostic factors in CRC. The conclusion of this research was based on a small sample size, and most of the patients were diagnosed with early colorectal carcinoma. Only 19 CRC patients had distant metastasis [20]. The independent prognostic value of the ApoB/ApoA-I in mCRC remains unknown. Therefore, we examined a large cohort of 838 patients and confirmed the independent prognostic value of the ApoB/ApoA-I in mCRC patients.

Numerous studies have indicated that ApoA-I and $A p o B$ play a non-negligible role in malignancies, but the underlying mechanisms are not fully known. Accumulating investigations revealed the potential mechanisms of these phenomena as follows: 1) According to several studies, ApoA-I is negatively associated with tumor-induced systemic inflammation, while elevated ApoB indicates a higher systemic inflammatory marker. Furthermore, a high ApoB/ApoA-I, which is considered atherogenic, may contribute to tumor necrosis[34, 35]; 2) A research group found that ApoA-I and its mimetic peptides can reduce the viability and proliferation of ovarian carcinoma cells in vivo and in vitro[36]; 3) Additionally, some studies indicated that apolipoprotein can initiate cellular signal transduction and promote antitumor drug delivery[14, 37, 38]; 4) In several studies, ApoA-I potently suppressed tumor growth and metastasis by both innate and adaptive immune processes[12, 13, $39,40]$.

The advantages of our study are described below. First, our study enrolled a large cohort of 838 CRC patients, and all the patients were diagnosed with mCRC. Second, we randomly assigned the patients into the training cohort and the validation cohort. To avoid a potential statistical bias, we also identified the independent prognostic value in a PSM cohort. The independent prognostic value of the ApoB/ApoA-I on OS in mCRC was confirmed in all three cohorts. We also validated our main findings and conclusions with an independent cohort. Third, we explored the prognostic value of the ApoB/ApoA-I and the LDL-C to HDL-C ratio (LHR) at the same time. The ApoB/ApoA-I was identified as an independent prognostic factor in all three cohorts, while LHR was identified as an independent prognostic factor in the training cohort only. However, there were several limitations to this study. First, all the patients were from a single cancer center, and all the patients were Chinese. The conclusion may not be suitable for Western populations. Second, the exact mechanism by which the ApoB/ApoA-I affects prognosis remains unknown. Third, although we used propensity score matching to reduce the imbalance in the baseline clinical variables, a selection bias might not have been completely avoided because of the retrospective nature of this research. 


\section{Conclusion}

In conclusion, the serum ApoB/ApoA-I is an independent prognostic factor for $O S$ in $m C R C$ patients. The prognostic value of the ApoB/ApoA-I should be further validated in a larger prospective and multicenter study.

\section{Abbreviations}

ApoA-I: apolipoprotein

A-I; ApoB: apolipoprotein $\mathrm{B} ; \mathrm{ApoB}$ to ApoA-I ratio: ApoB/ApoA-I; mCRC: metastatic colorectal cancer; ROC: receiver operating characteristic; PSM: propensity score matching; OS: overall survival; HR: hazard ratio; $\mathrm{CI}$ : confidence interval; MDT: multidisciplinary treatment; EMR: electronic medical record; IQR: interquartile range; ECOG PS score: Eastern Cooperative Oncology Group performance status score; CEA: serum carcinoembryonic antigen; CA19-9: carbohydrate antigen 19-9; HDL-C: high-density lipoprotein cholesterol; LDL-C: low-density lipoprotein cholesterol; LDL-C/HDL-C: LDL-C to HDL-C ratio.

\section{Acknowledgment}

This research was supported by the National Natural Science Foundation of China (81572392, 31501069, 81872011), Tip-top Scientific and Technical Innovative Youth Talents of Guangdong special support program (2016TQ03R614), the National Key R\&D Program of China (2018YFC1313300, 2016YFC1201704, 2017YFC1308900), Natural Science Foundation of Guangdong Province (2018A0303130282, 2018B030306049, 2017A030313485, 2014A030312015), Science and Technology Program of Guangdong (2015B020232008), China postdoctoral science foundation (2018M643301,2018M643340), Health \& Medical Collaborative Innovation Program of Guangzhou (201904020046, 201803040019, 201704020228) and Program for Guangdong Introducing Innovative and Entrepreneurial Teams (2017ZT07S096).

\section{Competing Interests}

The authors have declared that no competing interest exists.

\section{References}

1. Bray F, Ferlay J, Soerjomataram I, Siegel RL, Torre LA, Jemal A. Global cancer statistics 2018: GLOBOCAN estimates of incidence and mortality worldwide for 36 cancers in 185 countries. CA: a cancer journal for clinicians. 2018; 68: 394-424.

2. Siegel RL, Miller KD, Fedewa SA, Ahnen DJ, Meester RGS, Barzi A, et al. Colorectal cancer statistics, 2017. CA: a cancer journal for clinicians. 2017; 67: 177-93.

3. Zeng ZL, Luo HY, Yang J, Wu WJ, Chen DL, Huang P, et al. Overexpression of the circadian clock gene Bmal1 increases sensitivity to oxaliplatin in colorectal cancer. Clinical cancer research : an official journal of the American Association for Cancer Research. 2014; 20: 1042-52.
4. Borgquist S, Butt T, Almgren P, Shiffman D, Stocks T, Orho-Melander M, et al. Apolipoproteins, lipids and risk of cancer. International journal of cancer. 2016; 138: 2648-56.

5. Chandler PD, Song Y, Lin J, Zhang S, Sesso HD, Mora S, et al. Lipid biomarkers and long-term risk of cancer in the Women's Health Study. The American journal of clinical nutrition. 2016; 103: 1397-407.

6. Liao F, He W, Jiang C, Yin C, Guo G, Chen X, et al. A high LDL-C to HDL-C ratio predicts poor prognosis for initially metastatic colorectal cancer patients with elevations in LDL-C. OncoTargets and therapy. 2015; 8: 3135-42.

7. Melvin JC, Holmberg L, Rohrmann S, Loda M, Van Hemelrijck M. Serum lipid profiles and cancer risk in the context of obesity: four meta-analyses. Journal of cancer epidemiology. 2013; 2013: 823849.

8. Feng C, Ding G, Ding Q, Wen H. Overexpression of low density lipoprotein receptor-related protein 1 (LRP1) is associated with worsened prognosis and decreased cancer immunity in clear-cell renal cell carcinoma. Biochemical and biophysical research communications. 2018; 503: 1537-43.

9. Hu D, Peng F, Lin X, Chen G, Liang B, Chen Y, et al. Prediction of three lipid derivatives for postoperative gastric cancer mortality: the Fujian prospective investigation of cancer (FIESTA) study. BMC cancer. 2018; 18: 785.

10. Gilardoni MB, Remedi MM, Oviedo M, Dellavedova T, Sarria JP, Racca L, et al. Differential expression of Low density lipoprotein Receptor-related Protein 1 (LRP-1) and matrix metalloproteinase-9 (MMP-9) in prostate gland: From normal to malignant lesions. Pathology, research and practice. 2017; 213: 66-71.

11. dos Santos CR, Domingues G, Matias I, Matos J, Fonseca I, de Almeida JM, et al. LDL-cholesterol signaling induces breast cancer proliferation and invasion. Lipids in health and disease. 2014; 13: 16.

12. Hong Y, Manoharan I, Suryawanshi A, Shanmugam A, Swafford D, Ahmad S, et al. Deletion of LRP5 and LRP6 in dendritic cells enhances antitumor immunity. Oncoimmunology. 2016; 5: e1115941.

13. Zhang $\mathrm{H}$, Zhai $\mathrm{Z}$, Zhou H, Li $\mathrm{Y}, \mathrm{Li} \mathrm{X}$, Lin $\mathrm{Y}$, et al. Puerarin Inhibits oxLDL-Induced Macrophage Activation and Foam Cell Formation in Human THP1 Macrophage. BioMed research international. 2015; 2015: 403616.

14. Zhu WJ, Yang SD, Qu CX, Zhu QL, Chen WL, Li F, et al. Low-density lipoprotein-coupled micelles with reduction and $\mathrm{pH}$ dual sensitivity for intelligent co-delivery of paclitaxel and siRNA to breast tumor. International journal of nanomedicine. 2017; 12: 3375-93.

15. Han Y, Ding B, Zhao Z, Zhang H, Sun B, Zhao Y, et al. Immune lipoprotein nanostructures inspired relay drug delivery for amplifying antitumor efficiency. Biomaterials. 2018; 185: 205-18.

16. Ackerman D, Simon MC. Hypoxia, lipids, and cancer: surviving the harsh tumor microenvironment. Trends in cell biology. 2014; 24: 472-8.

17. Einbinder Y, Biron-Shental T, Agassi-Zaitler M, Tzadikevitch-Geffen K, Vaya J, Khatib S, et al. High-density lipoproteins (HDL) composition and function in preeclampsia. Archives of gynecology and obstetrics. 2018; 298: 405-13.

18. Marsche G, Saemann MD, Heinemann A, Holzer M. Inflammation alters HDL composition and function: implications for HDL-raising therapies. Pharmacology \& therapeutics. 2013; 137: 341-51.

19. Quan $\mathrm{O}$ Huang $\mathrm{Y}$, Chen $\mathrm{O}$, Oiu $\mathrm{H}, \mathrm{Hu} \mathrm{Q}$ Rong $\mathrm{Y}$, et al. Impact of Serum Apolipoprotein A-I on Prognosis and Bevacizumab Efficacy in Patients with Metastatic Colorectal Cancer: a Propensity Score-Matched Analysis. Transl Oncol. 2017; 10: 288-94.

20. Sirnio P, Vayrynen JP, Klintrup K, Makela J, Makinen MJ, Karttunen TJ, et al. Decreased serum apolipoprotein A1 levels are associated with poor survival and systemic inflammatory response in colorectal cancer. Scientific reports. 2017; 7: 5374.

21. Reddavide R, Misciagna G, Caruso MG, Notarnicola M, Armentano R, Caruso $\mathrm{ML}$, et al. Tissue expression of glycated apolipoprotein B in colorectal adenoma and cancer. Anticancer research. 2011; 31: 555-9.

22. Zhang X, Zhao XW, Liu DB, Han CZ, Du LL, Jing JX, et al. Lipid levels in serum and cancerous tissues of colorectal cancer patients. World journal of gastroenterology. 2014; 20: 8646-52.

23. Yang MH, Rampal S, Sung J, Choi Y-H, Son HJ, Lee JH, et al. The Association of Serum Lipids With Colorectal Adenomas. The American Journal of Gastroenterology. 2013; 108: 833-41.

24. Subbaiah PV, Chen $\mathrm{CH}$, Bagdade JD, Albers JJ. Substrate specificity of plasma lysolecithin acyltransferase and the molecular species of lecithin formed by the reaction. The Journal of biological chemistry. 1985; 260: 5308-14.

25. Van Hemelrijck M, Garmo H, Hammar N, Jungner I, Walldius G, Lambe M, et al. The interplay between lipid profiles, glucose, BMI and risk of kidney cancer in the Swedish AMORIS study. International journal of cancer. 2012; 130: 2118-28.

26. Van Hemelrijck M, Walldius G, Jungner I, Hammar N, Garmo H, Binda E, et al. Low levels of apolipoprotein A-I and HDL are associated with risk of prostate cancer in the Swedish AMORIS study. Cancer causes \& control : CCC. 2011; 22: 1011-9.

27. Akalanka HMK, Ekanayake S, Samarasinghe K. Could Anthropometric and Lipid Parameters Reflect Susceptibility to Breast Cancer? Comparison of Newly Diagnosed Breast Cancer and Apparently Healthy Women. Asian Pacific journal of cancer prevention : APJCP. 2018; 19: 2475-80.

28. Martin LJ, Melnichouk O, Huszti E, Connelly PW, Greenberg CV, Minkin S, et al. Serum lipids, lipoproteins, and risk of breast cancer: a nested case-control study using multiple time points. Journal of the National Cancer Institute. $2015 ; 107$. 
29. Guo S, He X, Chen Q, Yang G, Yao K, Dong P, et al. The Effect of Preoperative Apolipoprotein A-I on the Prognosis of Surgical Renal Cell Carcinoma: A Retrospective Large Sample Study. Medicine. 2016; 95: e3147.

30. Cheng T, Dai X, Zhou DL, Lv Y, Miao LY. Correlation of apolipoprotein A-I kinetics with survival and response to first-line platinum-based chemotherapy in advanced non-small cell lung cancer. Medical oncology. 2015; 32: 407.

31. Luo XL, Zhong GZ, Hu LY, Chen J, Liang Y, Chen OY, et al. Serum apolipoprotein A-I is a novel prognostic indicator for non-metastatic nasopharyngeal carcinoma. Oncotarget. 2015; 6: 44037-48.

32. Sacks FM. The crucial roles of apolipoproteins E and C-III in apoB lipoprotein metabolism in normolipidemia and hypertriglyceridemia. Current opinion in lipidology. 2015; 26: 56-63.

33. Yin Q, Chen X, Li L, Zhou R, Huang J, Yang D. Apolipoprotein $\mathrm{B}$ /apolipoprotein A1 ratio is a good predictive marker of metabolic syndrome and pre-metabolic syndrome in Chinese adolescent women with polycystic ovary syndrome. The journal of obstetrics and gynaecology research. 2013; 39: 203-9.

34. Walldius G, Jungner I. The apoB/apoA-I ratio: a strong, new risk factor for cardiovascular disease and a target for lipid-lowering therapy--a review of the evidence. Journal of internal medicine. 2006; 259: 493-519.

35. Faraj M, Messier L, Bastard JP, Tardif A, Godbout A, Prud'homme D, et al. Apolipoprotein B: a predictor of inflammatory status in postmenopausal overweight and obese women. Diabetologia. 2006; 49: 1637-46.

36. Su F, Kozak KR, Imaizumi S, Gao F, Amneus MW, Grijalva V, et al Apolipoprotein A-I (apoA-I) and apoA-I mimetic peptides inhibit tumor development in a mouse model of ovarian cancer. Proceedings of the National Academy of Sciences of the United States of America. 2010; 107: 19997-20002.

37. Zhao GJ, Yin K, Fu YC, Tang CK. The interaction of ApoA-I and ABCA1 triggers signal transduction pathways to mediate efflux of cellular lipids. Molecular medicine. 2012; 18: 149-58.

38. Feng J, Zhang J, Jackson AO, Zhu X, Chen H, Chen W, et al. Apolipoprotein A1 Inhibits the TGF-beta1-Induced Endothelial-to-Mesenchymal Transition of Human Coronary Artery Endothelial Cells. Cardiology. 2017; 137: 179-87.

39. Peng M, Zhang Q, Cheng Y, Fu S, Yang H, Guo X, et al. Apolipoprotein A-I mimetic peptide $4 \mathrm{~F}$ suppresses tumor-associated macrophages and pancreatic cancer progression. Oncotarget. 2017; 8: 99693-706.

40. Zamanian-Daryoush M, Lindner D, Tallant TC, Wang Z, Buffa J, Klipfell E, et al. The cardioprotective protein apolipoprotein A1 promotes potent anti-tumorigenic effects. The Journal of biological chemistry. 2013; 288: 21237-52. 